\title{
The spherical nucleic acids mRNA detection paradox
}

\author{
David Mason ${ }^{1,2}$, Gemma Carolan ${ }^{2}$, Marie Held ${ }^{2}$, Joan Comenge ${ }^{2}$, Sophie Cowman ${ }^{2}$, and Raphaël Lévy ${ }^{* 2}$ \\ ${ }^{1}$ Centre for Cell Imaging, Institute of Integrative Biology, University of Liverpool, Biosciences Building, Crown Street, \\ Liverpool L69 7ZB, UK \\ ${ }^{2}$ Department of Biochemistry, Institute of Integrative Biology, University of Liverpool, Biosciences Building, Crown Street, \\ Liverpool L69 7ZB, UK \\ *Corresponding author's e-mail address: rapha@liverpool.ac.uk
}

Published online: 17 November 2015 (Version 1); 29 March 2016 (version 2)

Cite as: Mason et al. ScienceOpen Research 2016 (DOI: 10.14293/S2199-1006.1.SOR-CHEM.AZ1MJU.v2)

Reviewing status: Please note that this article is under continuous review. For the current reviewing status and the latest referee's comments please click here or scan the QR code at the end of this article.

Primary discipline: Chemistry

Secondary discipline: Life sciences

Keywords: Gold nanoparticles, Smartflare, Nano-flare, Sticky-flare, Endosomal escape, Biological barriers, Open science, Live cell imaging, mRNA detection, Intracellular sensing

\section{ABSTRACT}

From the 1950s onwards, our understanding of the formation and intracellular trafficking of membrane vesicles was informed by experiments in which cells were exposed to gold nanoparticles and their uptake and localisation, studied by electron microscopy. In the last decade, building on progress in the synthesis of gold nanoparticles and their controlled functionalisation with a large variety of biomolecules (DNA, peptides, polysaccharides), new applications have been proposed, including the imaging and sensing of intracellular events. Yet, as already demonstrated in the 1950s, uptake of nanoparticles results in confinement within an intracellular vesicle which in principle should preclude sensing of cytosolic events. To study this apparent paradox, we focus on a commercially available nanoparticle probe that detects mRNA through the release of a fluorescently-labelled oligonucleotide (unquenching the fluorescence) in the presence of the target mRNA. Using electron, fluorescence and photothermal microscopy, we show that the probes remain in endocytic compartments and that they do not report on mRNA level. We suggest that the validation of any nanoparticle-based probes for intracellular sensing should include a quantitative and thorough demonstration that the probes can reach the cytosolic compartment.

The history of gold nanoparticles as tools for biological research and medical diagnostics starts more than a century ago. In 1912, Carl Friedrich August Lange invented a nanoparticle-based test to diagnose "dementia paralytica". The test was based on colour change upon aggregation induced by non-specific interactions with proteins from the spinal fluids of patients [1]. For several decades, the test was used in clinics and its existence spurred synthetic efforts towards better nanoparticle preparations [2]. With the development of electron microscopy in the 1940s and 1950s, gold nanoparticles became contrast agents of choice due to their high electron density, favourable dimensions and relative ease to prepare antibody conjugates. Some of the earliest studies of vesicular trafficking used gold colloids as electron microscopy probes. In 1957, Harford et al exposed HeLa cells to gold nanoparticles, observed localisation within intracellular vesicles by electron microscopy and concluded that their results supported the notion that "membranous organelles of the cytoplasm may be derived from the cell membrane" [3]. Thus, nanoparticles have been one of the early tools that biologists have used to decipher the way cells probe their environment. They were also used as a proxy to study the entry of biological nanoparticles, i.e. viruses, into cells. As early as 1965, it was noted in an elegant side-by-side electron microscopy study that while the Herpes viral particles escape endosomes, gold colloids remain in vesicles after uptake [4]. Indeed virions, being under a selective reproductive pressure, have evolved advanced means by which to escape their encapsulating vesicle and access the cytosol (for example, adenovirus [5]). Thus, more than five decades of work have clearly established that nanoparticles enter cells by endocytotic mechanisms that result in their entrapment inside intracellular vesicles unless those nanoparticles are biological in nature and have acquired through evolution, advanced molecular tools which enable them to escape. Access to the cytosol remains one of the main barriers towards biologics and siRNA therapeutic application [6-9]. For cell biology experiments, access to the cytosol can be gained through disruptive physical means such as electroporation or endosomal rupture through the use of osmotic pressure [10], liposome carriers or the proton sponge effect [11]. For purposes such as transfection, it is often acceptable that most particles remain trapped in endosomes as long as a few particles per cell reach the cell machinery. 
From the first decade of this century, the number of publications reporting the interaction and uptake of nanoparticles in cells has exploded, sometimes ignoring the knowledge gained from these earlier experiments. The idea that nanoparticles have a special capacity to cross biological barriers became pervasive, leading to concerns regarding their toxicity and hopes regarding their potential for therapeutic applications. Yet, both the concerns and the hopes appear to rest on limited data. The claim that nanoparticles can cross the cell membrane and access the cytosol seems to have entered the scientific literature through a 2005 article and a high-profile review published in 2006 by Geiser et al [12], and by Nel et al [13] respectively. The claim was challenged the following year by Shayla Banerji and Mark Hayes in an elegant experimental and theoretical study which was a direct response to the two papers cited above [14]. Unfortunately the refutation had a limited impact on the field. Thus, nanoparticles were proposed for intracellular sensing within the cytosol of live cells (without direct evidence of endosomal escape) including the detection of caspase three activity by plasmon rulers [15] and the regulation, quantification and imaging of specific mRNAs with spherical nucleic acids [16]. In both cases, the interpretation of the experiments relies on the particles being in the correct compartment: retention of the majority of the particles in endosomes would lead to incorrect conclusions.

RNA plays a central role in the conversion of DNA into proteins in cells. More than just a genetic translator, these highly specialised molecules play an active and highly dynamic role in gene expression, promoting silencing, up-regulation or modification of translation. Present studies on RNA are largely restricted to fixed or in vitro methods such as Fluorescent in situ Hybridisation (FISH), RT-PCR and gel electrophoresis. Fluorescent reporters that could get live, dynamic measurements of mRNA within cells or tissues would therefore have an enormous potential to improve our understanding of biology. The spherical nucleic acids gold nanoparticles developed by the Mirkin lab for detection of mRNA, are commercially available from EMD Merck Millipore under the name SmartFlares. The technology relies on the hybridisation of target mRNA strands with complementary strands which are covalently linked to a gold nanoparticle. Upon hybridisation a fluorescent reporter strand is displaced, and subsequently unquenched when no longer in the vicinity of the gold core, providing a readout of mRNA levels. A more recent implementation (named StickyFlares) relies on the same technology but inverts the sequences such that the fluorescent reporter strand is complementary to the mRNA of interest [17].

Given the limited amount of published information on the endosomal escape of spherical nucleic acids, we have studied the localisation of their commercially available form, i.e. SmartFlares. We have carried this research as an open science project, sharing our data and protocols in quasi-real time using an online notebook [18] and data repository [19]. This has allowed us to gather feedback from peers including other
SmartFlare users and developers. Using high-resolution fluorescence microscopy, electron microscopy and photothermal imaging we have found that using the manufacturers' instructions, the SmartFlares are taken up into vesicular compartments in a subset of cells. We find that after 16-20 hours, fluorescence is present and remains visible as puncta within membrane-contained vesicles. Importantly, the fluorescence levels are independent of the presence of the target mRNA. Given these findings, we suggest that the validation of any nanoparticle-based probes for intracellular sensing should include a quantitative and thorough demonstration that the probes can reach the cytosolic compartment.

\section{MATERIALS \& METHODS Materials}

SmartFlare mRNA detection probes were ordered from EMD Merck Millipore. Uptake and Scrambled controls were Cy5labelled while VEGF SmartFlares were Cy3-labelled. SmartFlares were re-suspended in $1 \mathrm{~mL}$ nuclease free de-ionised water and stored at room temperature protected from light according to the manufacturer's instructions.

Fluorescein isothiocyanate (FITC)-labelled $10 \mathrm{kDa}$ dextran (from Life Technologies - now ThermoFisher Scientific) was re-suspended to $10 \mathrm{mg} / \mathrm{mL}$ in phosphate-buffered saline (PBS). Dimethyloxaloylglycine (DMOG), Triton X-100 and Bovine Serum Albumin (BSA) were from Sigma Aldrich. DMOG stocks were used within 2 months of reconstitution and aliquoting into $-20^{\circ} \mathrm{C}$. All cell culture media was from Gibco (now ThermoFisher Scientific).

Primary antibodies were ordered from Abcam against LAMP1 (AB24170) and Transferrin Receptor (AB84036). Secondary antibodies were ordered from Life Technologies (now ThermoFisher Scientific) conjugated with AlexaFluor 488 (A11008), 568 (A11011) or 647 (A21244) as required.

HeLa cells (ATCC) were grown in $10 \mathrm{~cm}$ dishes (Corning) and split into $35 \mathrm{~mm}$ glass-bottomed dishes (Ibidi) for experiments.

\section{Cell culture}

HeLa cells were maintained in Dubecco's Modified Essential Medium (DMEM) growth media supplemented with 10\% Fetal Bovine Serum (FBS), 1\% non-essential amino acids and 1\% penicillin/streptomycin. Cells were maintained between 20 and $80 \%$ confluent at $37^{\circ} \mathrm{C}$ and $5 \% \mathrm{CO}_{2}$.

\section{SmartFlare mRNA detection experiments}

The SmartFlare RNA detection probes were used as directed by the manufacturer. Briefly; $\sim 4 \times 10^{5}$ HeLa cells were seeded onto $35 \mathrm{~mm}$ diameter glass-bottomed imaging dishes and incubated in growth media for 6 hours to adhere. Media were pre-mixed by aliquoting (per well) $960 \mu \mathrm{L}$ of media, $20 \mu \mathrm{L}$ of SmartFlare stock solution, $20 \mu \mathrm{L}$ of fluorescent dextran (10 mg/mL stock). In experiments with DMOG, the drug was included at $500 \mathrm{nM}$ and the volume of media 
reduced accordingly to compensate. Growth media was replaced with the above mixture and the cells returned to the incubator for $18 \mathrm{~h}$ or as noted.

\section{Quantitation of mRNA using qPCR}

Approximately $1 \times 10^{8}$ cells were seeded into $6 \mathrm{~cm}$ diameter dishes, allowed to adhere for 6 hours then treated with or without 500nM DMOG for 18 hours to replicate the SmartFlare experiments. Quantitative PCR was then performed as previously described [20]. Primers used were;

Cyclophillin A forward: GCTTTGGGTCCAGGAATGG

Cyclophillin A reverse: GTTGTCCACAGTCAGCAATGGT

VEGF forward: CTCCACCATGCCAAGTGGTC

VEGF reverse: GCAGTAGCTGCGCTGATAGA.

\section{Confocal microscopy}

Fluorescent imaging was conducted at the Centre for Cell Imaging (CCI) in Liverpool using a Zeiss LSM510 multiphoton microscope. Where possible, multi-pass dichroic mirrors and filters were used to reduce the potential for spatial misalignment. In all experiments, the confocal pinhole was adjusted to maintain a similar optical section between channels. For live experiments, incubation and $\mathrm{CO}_{2}$ control maintained environmental conditions as in 'Cell Culture' above. Unless otherwise stated, all fluorescent images are shown without any contrast enhancement.

\section{Electron microscopy}

Cells were fixed with a solution containing $1 \%$ paraformaldehyde and $3 \%$ gluteraldehyde in $0.1 \mathrm{M}$ cacodylate buffer ( $\mathrm{pH} 7.4$ ). They were stained first with reduced osmium $(2 \%$ OsO $+1.5 \%$ $\mathrm{K} 4[\mathrm{Fe}(\mathrm{CN}) 6])$. This was followed by a second osmium staining ( $2 \%$ Os04) and a uranyl acetate $(1 \%)$ staining. Samples were then dehydrated in graded ethanol $(30 \%, 50 \%, 70 \%, 90 \%$ and twice $100 \%)$. Finally, samples were infiltrated with medium TAAB resin 812 and embedded with the same resin. The resin was cured for $48 \mathrm{~h}$ at $60^{\circ} \mathrm{C}$. Ultrathin sections of $350 \mu \mathrm{m} \times 350 \mu \mathrm{m}$ $\times 74 \mathrm{~nm}$ were cut and placed in 200 mesh formvar/carbon filmed grids. They were post-stained with uranyl acetate and lead citrate before imaging on a Tecnai G3 spirit.

\section{Photothermal microscopy}

Cells were fixed in 4\% PFA for $20 \mathrm{~min}$ at room temperature. Subsequently, the cells were washed with PBS and immersed under $2 \mathrm{~mL}$ of PBS $+0.01 \%$ sodium azide for storage at $4^{\circ} \mathrm{C}$ between imaging sessions.

All images were acquired using a custom photothermal microscope [21] built around the body of a Nikon Eclipse Ti-U inverted microscope. Prior to photothermal imaging, the samples were allowed to equilibrate fully to room temperature for at least an hour. Fluorescence images were recorded using an X-Cite Series 120 light source (now Excelitas Technologies, USA) together with a FITC filter set for the Dextran signal and Cy5 filter set for the SmartFlare signal immediately prior to photothermal imaging.

The photothermal excitation laser $(523 \mathrm{~nm}$, frequencydoubled Nd:YAG, Ventus Laser Quantum, Germany) was tuned to $0.5 \mathrm{~mW}$ and modulated at a frequency of $459.5 \mathrm{kHz}$ using an accousto-optical modulator (Isomet Corporation, UK). The excitation beam was overlaid with a $1.0 \mathrm{~mW}$ non-resonant probe laser (633 nm, JDS Uniphase Corporation, USA) via a cold mirror (ThorLabs, USA). The superimposed beams were focused onto the sample via a Zeiss Plan Achromat $63 \times$ oil immersion objective (numerical aperture: 1.4). The sample was placed on a piezo scanning stage (MCL502385, MadCity Labs, USA), which allows the movement of the sample in three dimensions over the fixed laser spot. Pixel-by-pixel scanning was facilitated by a piezoelectric stage driver (MCL NanoDrive 85, MadCity Labs, USA) controlled through a Nanonis RC4 module and Nanonis program (SPECS Zurich, Switzerland). The transmitted and forward scattered light was collected via a Zeiss Achroplan 40× water immersion objective (numerical aperture: 0.8) and passed through a red-pass filter (ThorLabs, USA) to block the excitation laser. The red component was focused on a photodiode of the balanced photo receiver (Model $210710 \mathrm{MHz}$ adjustable photo receiver, New Focus, USA). A lock-in amplifier (DSP 7260, Signal Recovery, USA) was used to identify the scattered component of the probe beam that corresponds to the modulation frequency (i.e. $459.5 \mathrm{kHz}$ ). A Nanonis SC4 Acquisition Module (SPECS Zurich, Switzerland) was used for signal acquisition. The signal was averaged and a grayscale pixel value was generated. The pixel-by-pixel values were then converted into a photothermal image and saved in the Nanonis-native .sxm format.

\section{Immunofluorescence}

Cells were treated identically as to the " $m R N A$ detection experiments" above, however instead of imaging the samples live, they were washed once in Phosphate Buffered Saline (PBS), then fixed in 4\% PFA for $20 \mathrm{~min}$ at room temperature. Subsequently, samples were washed at least once with PBS to remove excess fixative then blocked and permeabilised using $0.1 \%$ TritonX-100 plus 1\% Bovine Serum Albumin (BSA) in PBS for 60 minutes at room temperature. Samples were washing three times with PBS. Primary antibodies were diluted in 1\% BSA in PBS at 1:500, and added to the samples for 60 mins at room temperature. Samples were washed three times for 15 mins each in PBS, then secondary antibodies were added at 1:1000 dilution in 1\% BSA in PBS for 60 mins at room temperature. Cells were washed three times in PBS then imaged immediately or stored under $2 \mathrm{~mL}$ of PBS + $0.01 \%$ sodium azide at $4^{\circ} \mathrm{C}$ for later imaging. 

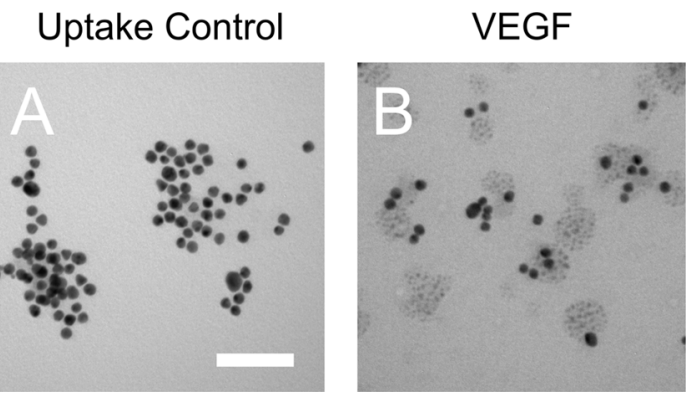

Figure 1. Physical Characterisation of SmartFlare RNA detection probes: SmartFlares were re-suspended in nuclease-free deionised water and $10 \mu \mathrm{L}$ dried onto formvar/carbon electron microscopy grids. Samples of the Uptake Control (A) and VEGF SmartFlares (B) were imaged on a Tecnai spirit Transmision Electron Microscope (TEM) at a nominal magnification of 150,000 times. The scale bar represents $100 \mathrm{~nm}$.

\section{Data analysis}

All data analysis was performed using Fiji (available from http://fiji.sc). In Figure 1, estimates of particle size were made on calibrated images by thresholding the electron dense particles by intensity, followed by the application of a $2 \mathrm{D}$ watershed filter to split separating objects. The Analyze Particles function was used to report the minimum and maximum Feret diameters of particles with an area greater than $100 \mathrm{~nm}^{2}$ with circularity greater than 0.7 (to exclude amorphous bunches of particles). For each particle, the mean of the maximum and minimum Feret diameter were calculated. The population mean is reported.

In Figure 3, the change in intensity after DMOG treatment was measured by background subtracted raw integrated density for a field of cells and dividing this by the number of cells in the field. Within experimental repeats, the data were normalised to an untreated control, and the mean of these values were reported. The results of three experiments (each with 8 fields) were analysed in this way. A two-tailed heteroscedastic Student's t-test was used to assess significance $(\mathrm{P}=0.065)$.

In Figure 4, the SmartFlare fluorescence channel was compared to a second channel (see Figure for details) and analysed using the JaCoP plugin for Fiji [22], for colocalisation using a thresholded Manders' analysis. Reported (yellow number) is the mean of 12 fields (approximately 20 cells per field) from two independent experiments (TFN-R \& LAMP1) or 24 fields from two separate experiments (dextran). Given the non-homogeneous uptake of SmartFlares by cells, the values reported are the coefficient relating to the overlap between SmartFlares and the secondary label.

\section{Open science}

This project has been run as an Open Science project, with results, thoughts, commentary and discussion posted at [http://raphazlabcommons.wordpress.com]. The raw data were both managed internally and made available, through the OMERO server at the Liverpool Centre for Cell Imaging (CCI). We would like to acknowledge the help of the Open Microscopy Environment group (University of Dundee), specifically Will Moore, for help with developing our public-facing gallery hosted at [http://cci02.liv.ac.uk/gallery].

\section{RESULTS AND DISCUSSION Characterisation of SmartFlare RNA detection probes}

As an exemplar target, we chose the Vascular Endothelial Growth Factor (VEGF) because it is present in all mammalian cells, its regulation is relevant to a range of diseases, and its expression level can be manipulated pharmacologically with dimethyloxalylglycine (DMOG) [23,24].

As gold nanoparticles have a known propensity to aggregate and agglomerate, we sought to first characterise the VEGF SmartFlares along with the 'Uptake Control'. Transmission electron microscopy (TEM) showed no apparent aggregation (Figure $1 \mathrm{~A} \& \mathrm{~B}$ ) of the particles and allowed a measurement of the particle diameters (VEGF: $16 \mathrm{~nm}, \mathrm{SD}=2.34 \mathrm{~nm}, \mathrm{n}=310$ particles; uptake control: $14 \mathrm{~nm}, \mathrm{SD}=1.82 \mathrm{~nm}, \mathrm{n}=93$ particles) which compared well to the expected diameter of $13 \mathrm{~nm}$.

\section{SmartFlares are taken up into cells}

Following the manufacturers' instructions, Uptake Control SmartFlares were added to HeLa cells and incubated for 18 hours. Live-cell imaging with a laser-scanning confocal microscope revealed fluorescent puncta of $\sim 1 \mu \mathrm{m}$ diameter (Figure $2 \mathrm{~A}$ and the project's online data repository at [http://cci02.liv.ac.uk/ gallery]). Surprisingly, not all cells appeared to internalise the Uptake Control SmartFlares, as evidenced by a lack of detectable fluorescent signal in some cells (Figure 2C). Both the VEGF and Scrambled SmartFlares showed a very similar punctate distribution within the cells. This was unexpected for two main reasons. Firstly, SmartFlares are sold on the basis of being able to escape the endosomes in order to interact with RNA in the cytosol $[25,26]$. Secondly, in order to become fluorescent, the SmartFlares are ostensibly required to interact with target RNA molecules, of which we would expect limited amounts within newly formed endocytic vesicles.

\section{SmartFlares remain in vesicles up to $18 \mathrm{~h}$}

The punctate distribution seen in Figure 2 could result from aggregation in the cytosol, vesicular containment or binding to the surface of cells. In order to examine this at higher resolution, SmartFlare-loaded cells were imaged using transmission electron microscopy (TEM). From an initial screen of the EM grid studying at least 20 cells, gold particles were only seen to be contained within membrane-bound compartments (Figure 3A-B). Some sections did not present any gold nanoparticles.

The TEM data show that the SmartFlares are contained within membrane-bound compartments. To test whether their fluorescence is nevertheless sensitive to the levels of VEGF mRNA, cells were treated with $500 \mathrm{nM}$ DMOG at the time of 


\section{SmartFlare}
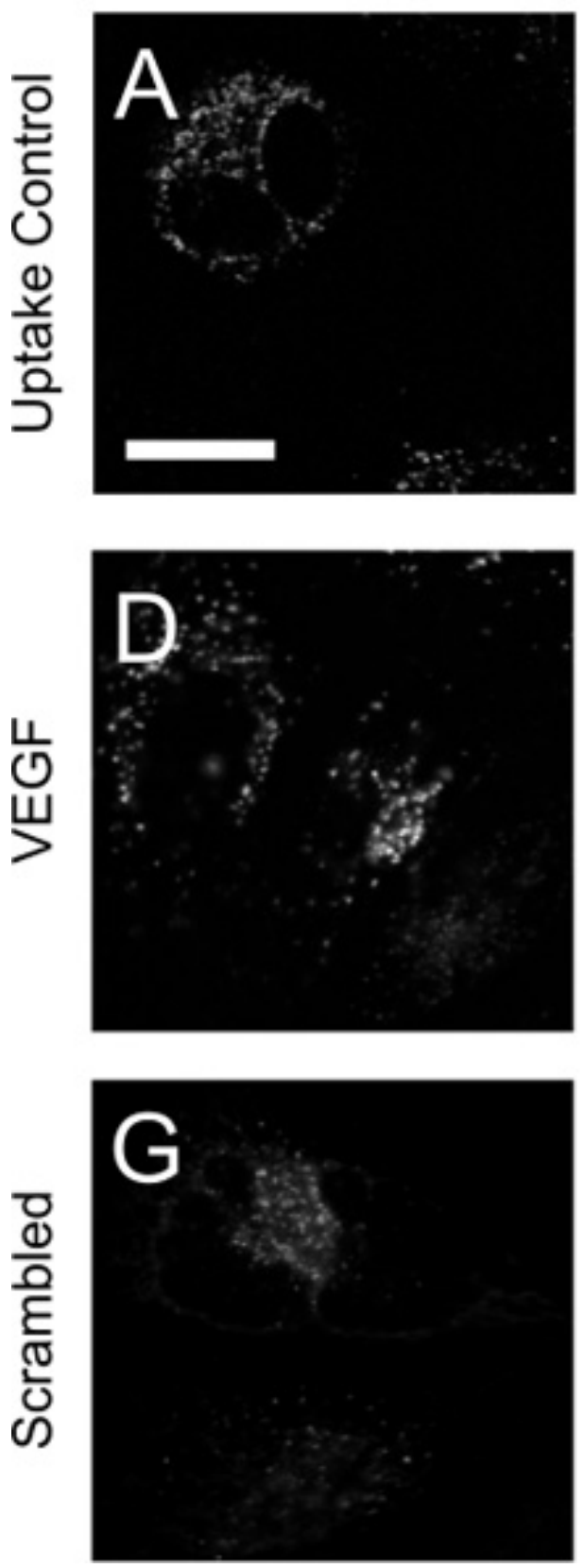

Transmitted
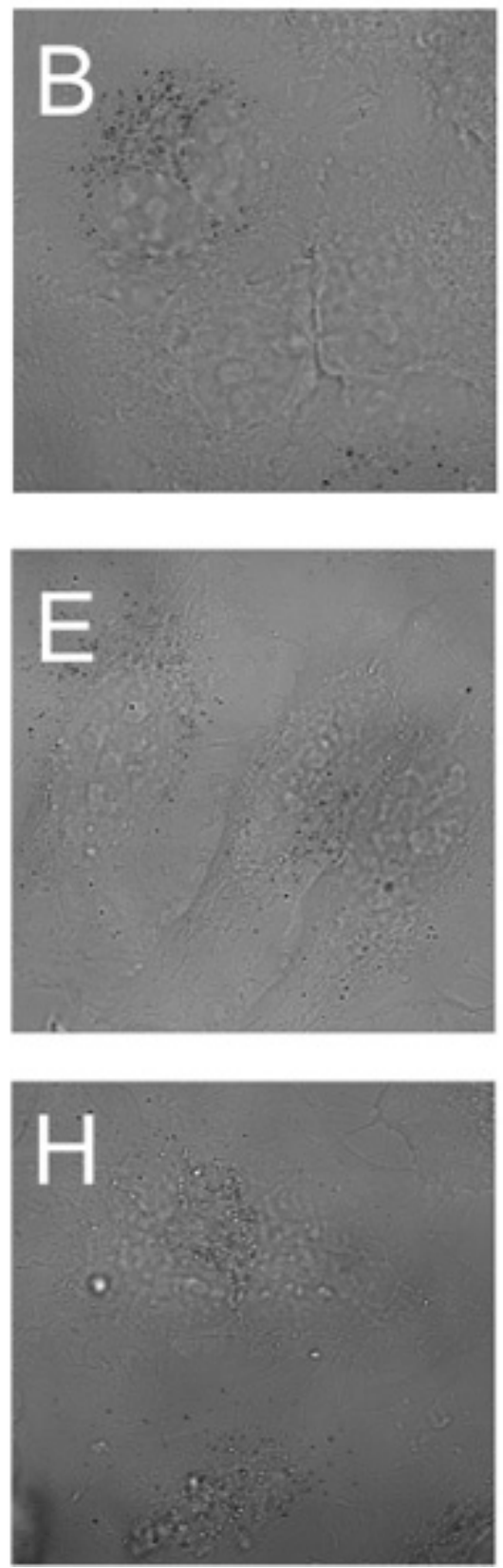

Merge
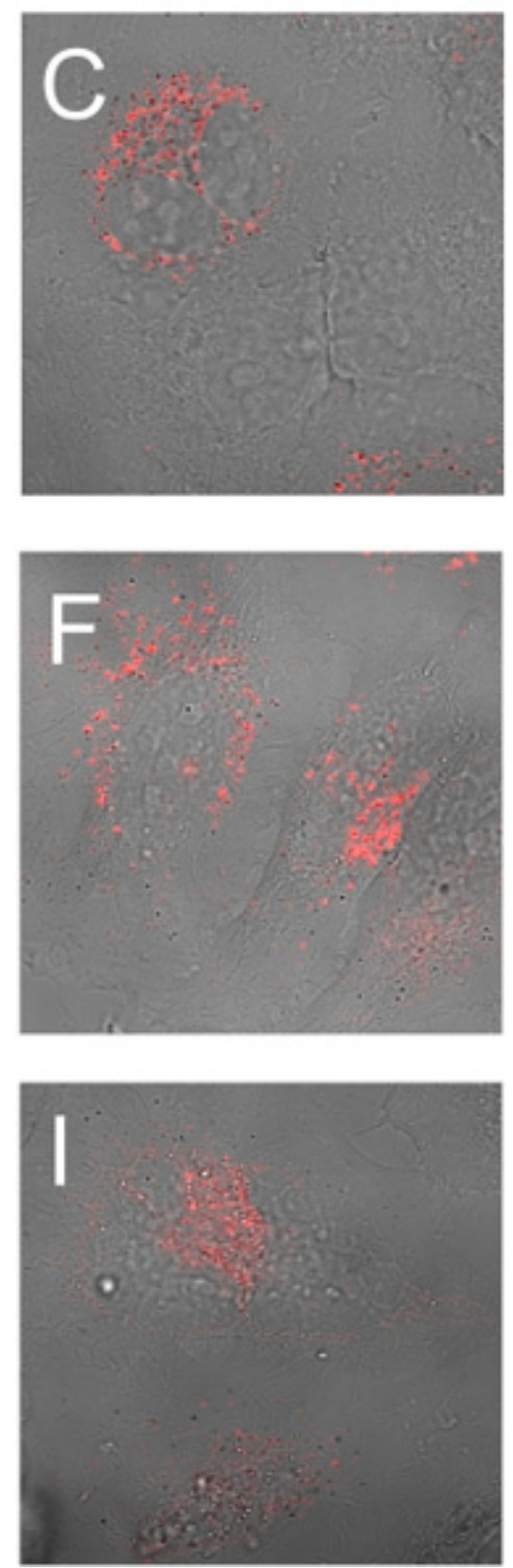

Figure 2. Cellular Uptake of SmartFlares: HeLa cells were exposed to Uptake Control (A-C), VEGF (D-E) or Scrambled Control (G-I) SmartFlares for 18 hours, then washed and imaged with confocal laser-scanning microscopy in fluorescence $(A, D, G)$ or transmitted $(\mathrm{B}, \mathrm{E}, \mathrm{H})$ channels. An overlay of the two channels is shown in $\mathrm{C}, \mathrm{F}$ and I. All frames are shown at identical magnification, scale bar represents $10 \mu \mathrm{m}$.

SmartFlare addition. Previous studies have shown DMOG to increase VEGF mRNA levels by 10 fold in different cell types [27]. Here, DMOG increased VEGF mRNA on average 19 fold $(21,15$ and 22 fold in three independent experiments). As shown in Figure 3C-E however, 18 hours of DMOG treatment did not affect the intensity of the VEGF SmartFlare signal.
To evaluate the respective localisation of the gold core and unquenched fluorescence reporter, we combined fluorescence and photothermal microscopy to visualise the gold core and fluorescent dye in the same sample. This showed the fluorescent puncta and nanoparticles to be within the same compartments (Figure 3F-H). 


\section{Uptake Control SmartFlare}

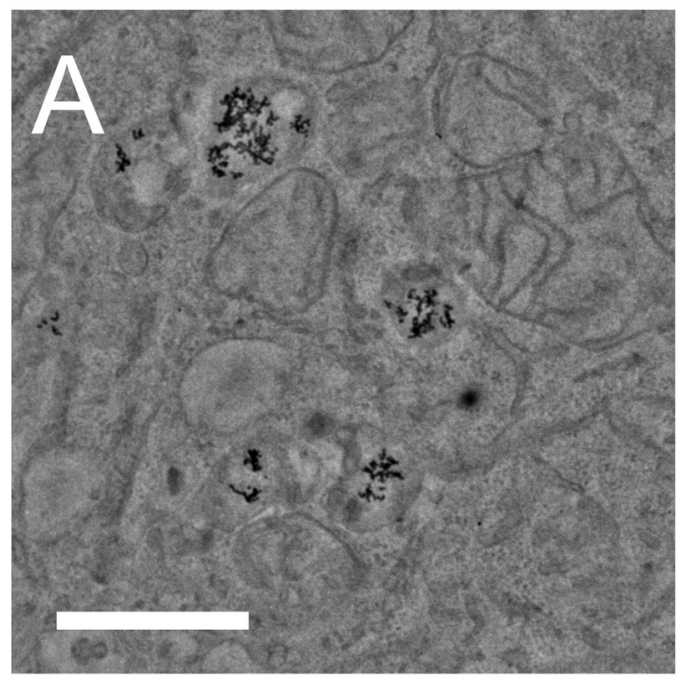

VEGF SmartFlare

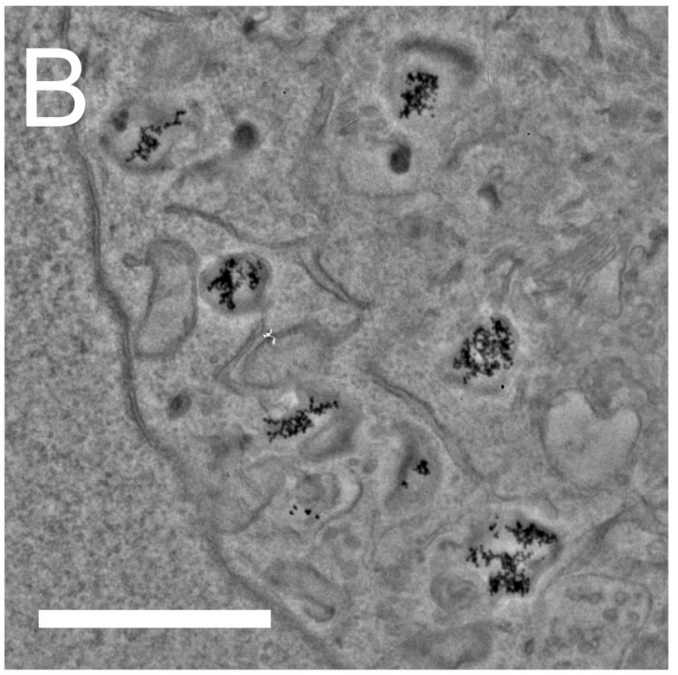

\section{VEGF SmartFlare}

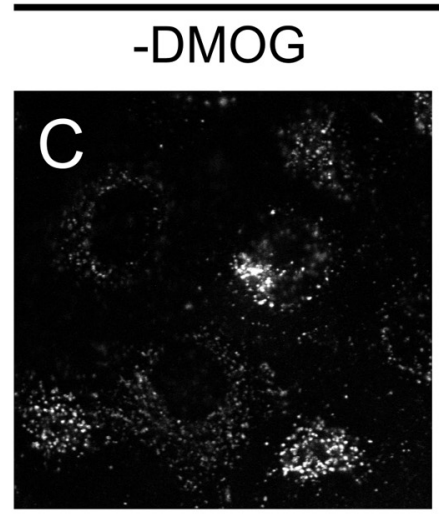

+ DMOG
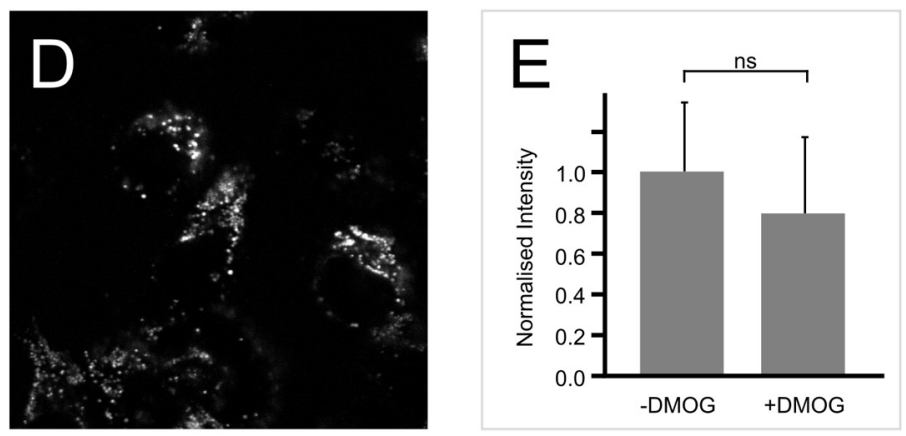

\section{SmartFlare}

\section{Fluorescence}
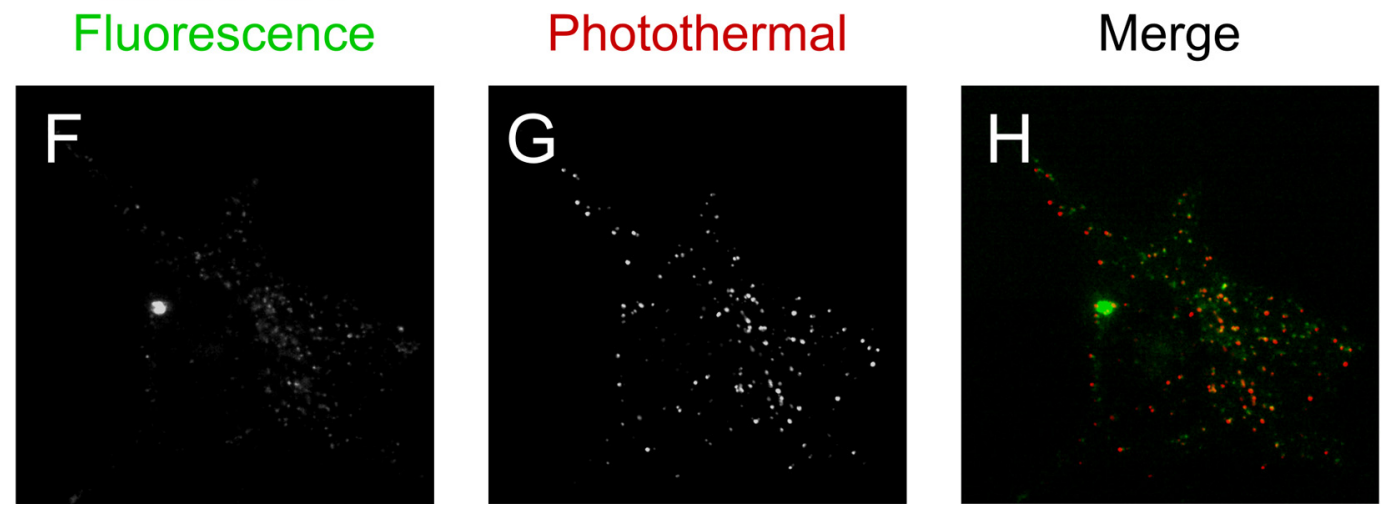

Figure 3. Confirming Vesicular Containment of SmartFlares: HeLa cells were loaded with Uptake Control (A) or VEGF (B) SmartFlares for 18 hours then prepared for TEM as detailed in methods. Scale bars represent $1000 \mathrm{~nm}$. Confocal microscopy was used to study VEGF SmartFlare-loaded cells (C) and cells treated with $500 \mathrm{nM}$ DMOG for 18 hours (D). No increase in intensity was observed in three independent experiments (E). Correlative fluorescence (F) and photothermal (G) microscopy was used to view SmartFlare fluorescence and gold nanoparticle cores in the same sample. 

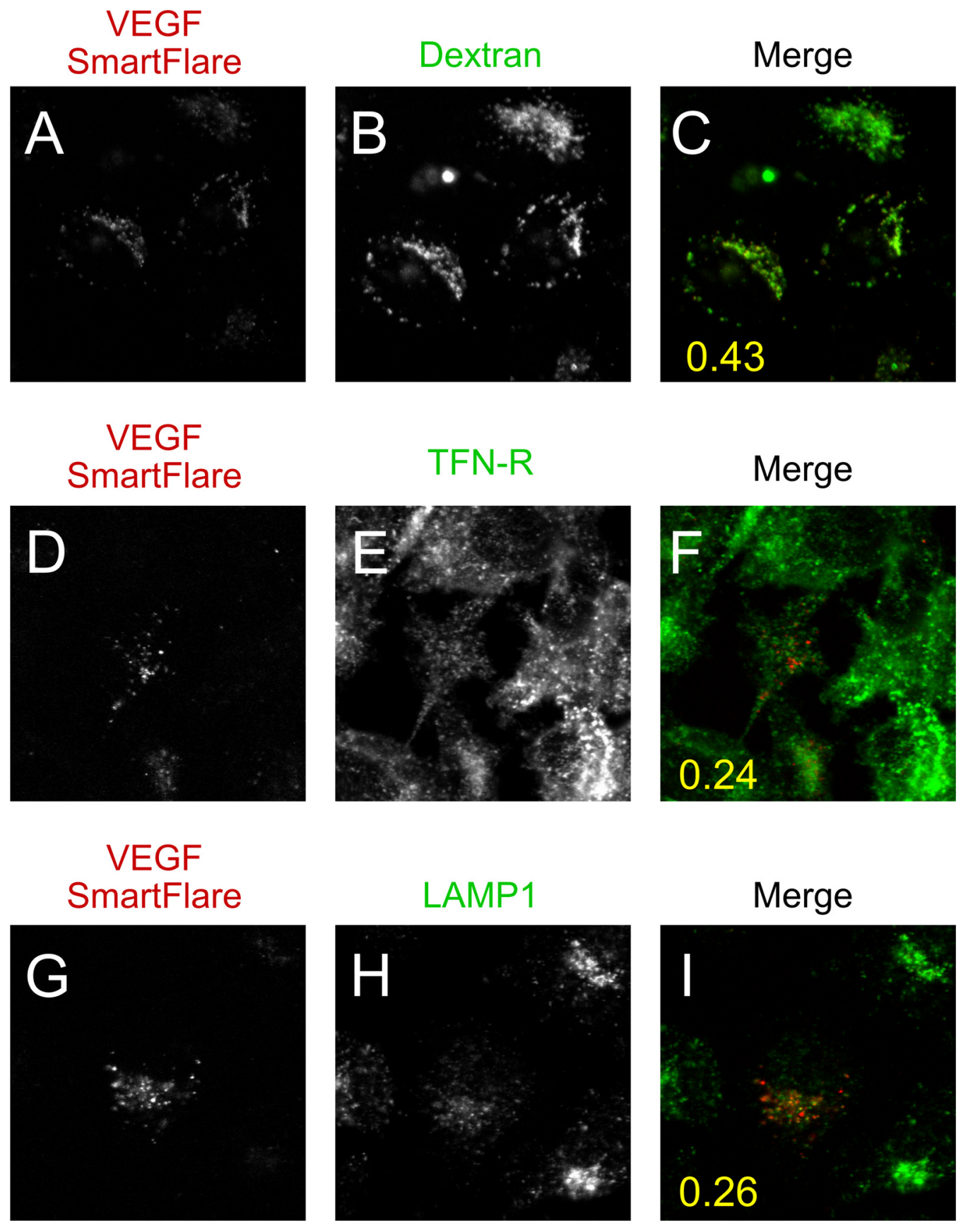

Figure 4. Studying the localisation of SmartFlares: HeLa cells were loaded with the VEGF SmartFlares in combination with a fluorescent $10 \mathrm{kDa}$ dextran to label fluid-phase endocytic pathways (A-C). After fixation, samples were immunostained either for the Transferrin Receptor (TFN-R: D-F) or Lysosome-associated Membrane Protein 1 (LAMP1: G-I) to demark the recycling and lysosomal compartments respectively. Shown are SmartFlare fluorescence $(A, D, G)$ and dextran or secondary antibody fluorescence $(B, E, H)$. Panels $(C, F, I)$ show an overlay of the two channels (SmartFlares pseudo-coloured red) with the mean Manders' coefficient (yellow number) expressing the proportion of SmartFlare Signal overlapping with the respective marker for all fields analysed.

\section{Characterisation of the SmartFlare-containing vesicles}

Having confirmed that the SmartFlares were membranebound, we set about trying to characterise the SmartFlarecontaining compartments. There are several ways in which material impermeant to the cell membrane can be taken up into cells. Endocytosis is probably the most well studied category and is largely driven by receptor-mediated signalling, whereas pinocytic processes are considered constitutive and are responsible for fluid-phase (IE receptor independent) uptake. The terminal compartment of these processes 
is the lysosome, a highly nucleolytic and proteolytic compartment involved in degradation and in some cells, antigen presentation.

We labelled the endocytic pathway using a fluorescentlylabelled $10 \mathrm{kDa}$ dextran, both to show that constitutive uptake was occurring in our system, but also to label all of the compartments of the pathway. As expected, the dextran labelled every cell in the field with homogeneous intensity puncta (Figure 4B). There were approximately the same number of vesicles per cell when corrected for cell size. Interestingly, the dextran and SmartFlares showed a Manders' colocalisation coefficient of 0.43 (Figure 4C: see Methods for details). Furthermore, much the same was seen at a 2 hour time point (Supplementary Figure 1).

As the dextran may be excluded from receptor-mediated endosomes (by unknown mechanisms) we also used immunofluorescence to label the recycling (transferrin-receptor positive) and terminal (lysosomal) compartments of the endocytic pathway (Figure 4D-F and G-I respectively). Upon analysis, the SmartFlares showed little overlap with either of these compartments (Figure 4C,F,I, mean Manders' coefficient of at least 12 fields from two independent experiments is inset), suggesting a parallel but largely non-overlapping compartment.

\section{CONCLUSIONS}

Developing the Green Fluorescent Protein (GFP) into a tool for researchers was a landmark achievement across many fields and has opened up the dynamic study of proteins in living cells [28]. There is no doubt that replicating this success in the study of RNA would be equally as momentous. Our results however, indicate that SNAs in their present incarnation, cannot be used to report on mRNA levels in live cells. Furthermore, we have shown that SNAs are taken up into only a subset of cells (which could potentially bias population studies). Once taken up we consistently observe a punctate distribution indicating retention within vesicular compartments. This was confirmed by electron microscopy and photothermal imaging. Furthermore, the controls (the scrambled and constitutively-fluorescent uptake control) showed similar levels of fluorescence. As the fluorophore should only be unquenched in the presence of cognate mRNA, which we assume is not present in the vesicles, this signal is likely the result of a nucleolytic cleavage of the oligonucleotide strands. Indeed the Mirkin group themselves have published a comprehensive study on the vesicular retention, localisation and degradation of SNAs, particularly highlighting the role of DNaseII [29].

The same study highlights what an effective tool this must be if only a "...small, unquantifiable portion of these particles escape the endosome..." (ibid). Even if we accept the possibility of a small fraction of these particles being released, unscathed into the cytosol, we still have the issue of overcoming background signal. StickyFlares (and presumably by extension, SmartFlares) are reported to increase in fluorescence intensity ten times upon un-quenching [17], which means that even if as many as one in eleven are released, the signal would not be visible over the background fluorescence. All of this is ignoring cleavage and unquenching inside of the vesicle, which would further increase the background.

These findings leave us with the difficult job of interpreting the existing body of work published using these probes. It is likely that publication bias is playing a role: we do not know how many laboratories have bought and tried the SmartFlare reagents versus how many have published their results. Most of the published SmartFlare data are cytometry results which do not give any indication of the cellular localisation of the signal. Interestingly, when imaging data are included they show a punctate distribution in cells [30-32] although this is not commented upon. There have been other cases, most notably that of antisense technology, working but having an effect through means other than traditional Watson-Crick base pairing [33]. Thus, in addition to confirmation bias, some of the published work may result from poorly understood off-target effects.

\section{REFERENCES}

[1] Cruickshank J. The value and mechanism of the colloidal gold test. Br J Exp Pathol. 1920;1:71-88.

[2] Patterson J. The preparation and standardization of colloidal gold for the Lange test. Br J Exp Pathol. 1931;12:143-6.

[3] Harford CG, Hamlin A, Parker E. Electron microscopy of HeLa cells after the ingestion of colloidal gold. J Biophys Biochem Cytol. 1957;3:749-56. doi:10.1083/jcb.3.5.749

[4] Epstein MA, Hummeler K, Berkaloff A. The entry and distribution of herpes virus and colloidal gold in HeLa cells after contact in suspension. J Exp Med. 1964;119:291-302. doi:10.1084/ jem.119.2.291

[5] Maier O, Marvin SA, Wodrich H, Campbell EM, Wiethoff, CM. Spatiotemporal dynamics of adenovirus membrane rupture and endosomal escape. J Virol. 2012;86:10821-8. doi:10.1128/JVI. 01428-12

[6] Bogart LK, Pourroy G, Murphy CJ, Puntes V, Pellegrino T, Rosenblum D, Peer D, Lévy R. Nanoparticles for imaging, sensing, and therapeutic intervention. ACS Nano. 2014;8:3107-22. doi:10.1021/nn500962q

[7] Iversen TG, Skotland T, Sandvig K. Endocytosis and intracellular transport of nanoparticles: present knowledge and need for future studies. Nano Today. 2011;6:176-85. doi:10.1016/j. nantod.2011.02.003

[8] Gilleron J, Querbes W, Zeigerer A, Borodovsky A, Marsico G, Schubert U, Manygoats K, Seifert S, Andree C, Stöter M, et al. Image-based analysis of lipid nanoparticle-mediated siRNA delivery, intracellular trafficking and endosomal escape. Nat Biotechnol. 2013;31:638-46. doi:10.1038/nbt.2612

[9] Varkouhi AK, Scholte M, Storm G, Haisma HJ. Endosomal escape pathways for delivery of biologicals. J Control Release. 2011;151:220-8. doi:10.1016/j.jconrel.2010.11.004

[10] Okada CY, Rechsteiner M. Introduction of macromolecules into cultured mammalian cells by osmotic lysis of pinocytic vesicles. Cell. 1982;29:33-41. doi:10.1016/0092-8674(82)90087-3

[11] Martens TF, Remaut K, Demeester J, De Smedt SC, Braeckmans $\mathrm{K}$. Intracellular delivery of nanomaterials: how to catch endosomal escape in the act. Nano Today. 2014;9:344-64. doi:10.1016/ j.nantod.2014.04.011 
[12] Geiser M, Rothen-Rutishauser B, Kapp N, Schürch S, Kreyling W, Schulz H, Semmler M, Im Hof V, Heyder J, Gehr P. Ultrafine particles cross cellular membranes by nonphagocytic mechanisms in lungs and in cultured cells. Environ. Health Perspect. 2005;113:1555-60. doi:10.1289/ehp.8006

[13] Nel A, Xia T, Mädler L, Li N. Toxic potential of materials at the nanolevel. Science. 2006;311:622-7. doi:10.1126/science.1114397

[14] Banerji SK, Hayes MA. Examination of Nonendocytotic Bulk Transport of Nanoparticles Across Phospholipid Membranes. Langmuir. 2007;23:3305-13. doi:10.1021/la0622875

[15] Jun Y, Sheikholeslamia S, Hostetterb DR, Tajonb C, Craik CS, Paul Alivisatosa A. Continuous imaging of plasmon rulers in live cells reveals early-stage caspase-3 activation at the singlemolecule level. Proc Natl Acad Sci U S A. 2009;106:17735-40. doi:10.1073/pnas.0907367106

[16] Seferos DS, Giljohann DA, Hill HD, Prigodich AE, Mirkin CA. Nanoflares: probes for transfection and mRNA detection in living cells. J Am Chem Soc. 2007;129: 15477-9. doi:10.1021/ja0776529

[17] Briley WE, Bondy MH, Randeria PS, Dupper TJ, Mirkin CA. Quantification and real-time tracking of RNA in live cells using Sticky-flares. Proc Natl Acad Sci U S A. 2015;112:9591-5. doi:10.1073/pnas.1510581112

[18] Mason D, Carolan G, Comenge J, Held M, Levy R. Posts about SmartFlare on Rapha-z-lab-commons [cite 2014 Sep]. Available from https://raphazlabcommons.wordpress.com/tag/smartflare/

[19] SmartFlares data repository [cite 2014 Sep]. Available from http://cci02.liv.ac.uk/gallery/show_project/1217/

[20] Richards R, Jenkinson MD, Haylock BJ, See V. Cell cycle progression in glioblastoma cells is unaffected by pathophysiological levels of hypoxia. PeerJ 2016:4, e1755.

[21] Nieves DJ, Li Y, Fernig DG, Lévy R. Photothermal raster image correlation spectroscopy of gold nanoparticles in solution and on live cells. R Soc Open Sci. 2015;2:140454. doi:10.1098/ rsos. 140454

[22] Bolte S, Cordelières FP. A guided tour into subcellular colocalization analysis in light microscopy. J Microsc. 2006;224:213-32. doi:10.1111/j.1365-2818.2006.01706.x

[23] Zhu T, Park HC, Son KM, Yang HC. Effects of dimethyloxalylglycine on wound healing of palatal mucosa in a rat model. BMC Oral Health. 2015;15:60. doi:10.1186/s12903-015-0047-1

[24] Dehne N, Kerkweg U, Otto T, Fandrey J. The HIF-1 response to simulated ischemia in mouse skeletal muscle cells neither enhances glycolysis nor prevents myotube cell death. Am J Physiol Regul Integr Comp Physiol. 2007;293:R1693-701. doi:10.1152/ ajpregu.00892.2006

[25] EMD Millipore Bioscience. What if you could detect RNA in LIVING CELLS? 2014 [cite 2014 Sep]. Available from https:// www.youtube.com/watch?v=CiSC2B8hl1E

[26] EMD Millipore Bioscience. SmartFlareTM Live Cell RNA Detection [cite 2014 Sep]. Available from http://www.merckmillipore.com/INTERSHOP/web/WFS/Merck-GB-Site/en_US/-/GBP/ ViewParametricSearch-SimpleOfferSearch?search=\&SearchTerm= smartflare

[27] Herrmann A, Rice M, Lévy R, Pizer BL, Losty PD, Moss D, Sée V. Cellular memory of hypoxia elicits neuroblastoma metastasis and enables invasion by non-aggressive neighbouring cells. Oncogenesis. 2015;4:e138. doi:10.1038/oncsis.2014.52

[28] Enterina JR, Wu L, Campbell RE. Emerging fluorescent protein technologies. Curr Opin Chem Biol. 2015;27:10-17. doi:10.1016/j.cbpa.2015.05.001

[29] Wu XA, Choi CHJ, Zhang C, Hao L, Mirkin CA. Intracellular fate of spherical nucleic acid nanoparticle conjugates. J Am Chem Soc. 2014;136:7726-33. doi:10.1021/ja503010a

[30] Lahm H, Doppler S, Dreßen M, Werner A, Adamczyk K, Schrambke D, Brade T, Laugwitz KL, Deutsch MA, Schiemann $\mathrm{M}$, et al. Live fluorescent RNA-based detection of pluripotency gene expression in embryonic and induced pluripotent stem cells of different species. Stem Cells. 2015;33:392-402. doi:10.1002/stem.1872

[31] Kratz A, Beguin P, Kaneko M, Chimura T, Suzuki AM, Matsunaga A, Kato S, Bertin N, Lassmann T, Vigot R, et al. Digital expression profiling of the compartmentalized translatome of Purkinje neurons. Genome Res. 2014;24:1396-410. doi:10.1101/gr. 164095.113

[32] Krönig M, Walter M, Drendel V, Werner M, Jilg CA, Richter AS, Backofen R, McGarry D, Follo M, Schultze-Seemann W, et al. Cell type specific gene expression analysis of prostate needle biopsies resolves tumor tissue heterogeneity. Oncotarget. 2015; 6:1302-14. doi:10.18632/oncotarget.2744

[33] Raffo A. Antisense RNA down-regulation of bcl-2 expression in DU145 prostate cancer cells does not diminish the cytostatic effects of G3139 (Oblimersen). Clin Cancer Res. 2004;10: 3195-206. doi:10.1158/1078-0432.CCR-03-0287

\section{COMPETING INTERESTS}

The authors declare no competing interests.

\section{PUBLISHING NOTES}

(C) 2016 Mason et al. This work has been published open access under Creative Commons Attribution License CC BY 4.0, which permits unrestricted use, distribution, and reproduction in any medium, provided the original work is properly cited. Conditions, terms of use and publishing policy can be found at www.scienceopen.com.

Please note that this article may not have been peer reviewed yet and is under continuous post-publication peer review. For the current reviewing status please click here or scan the QR code on the right.

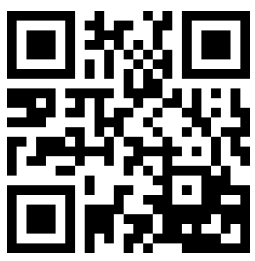

\section{scienceOPEN.com}

research+publishing network 


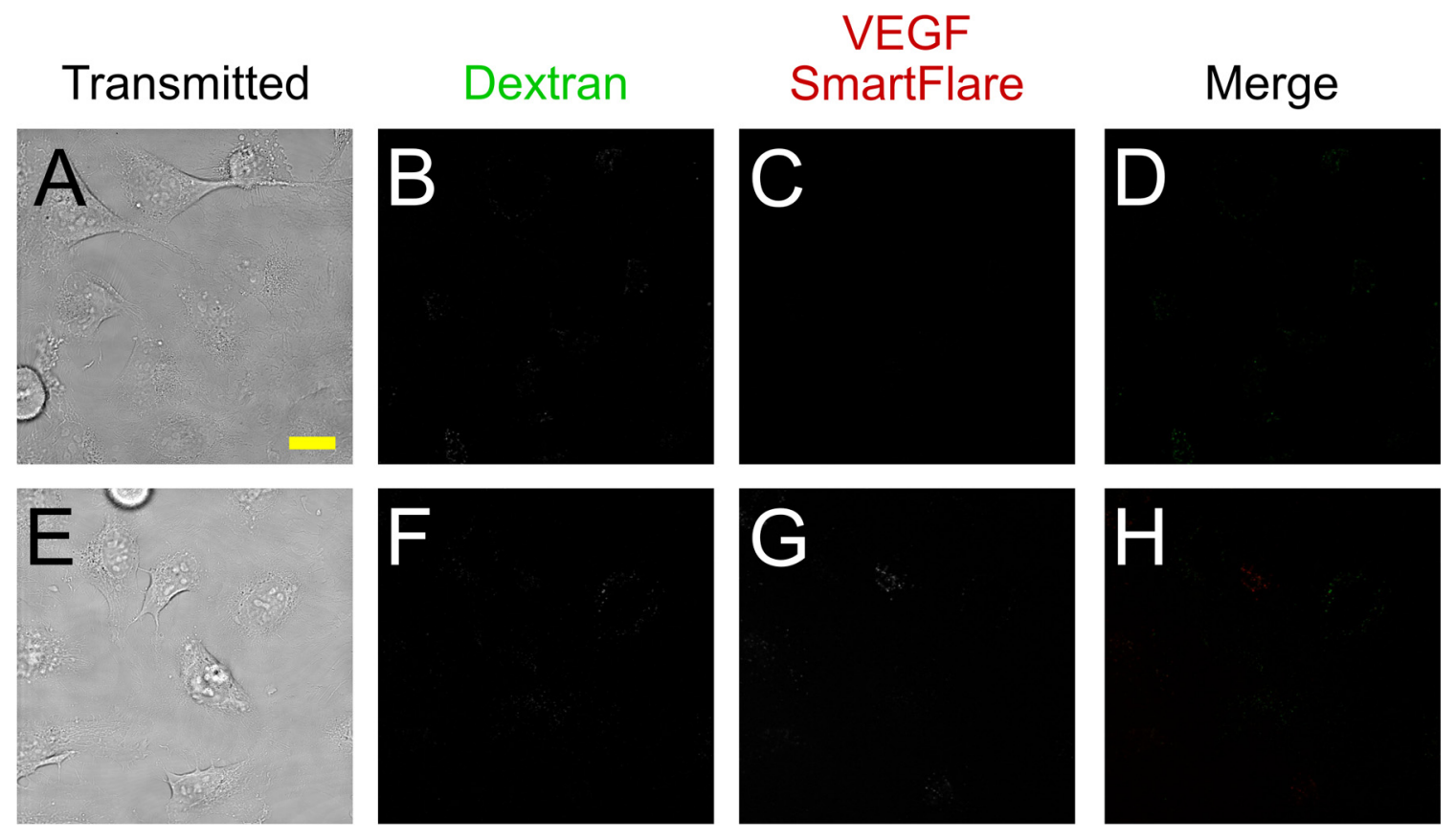

Same acquisition settings as Figure 2
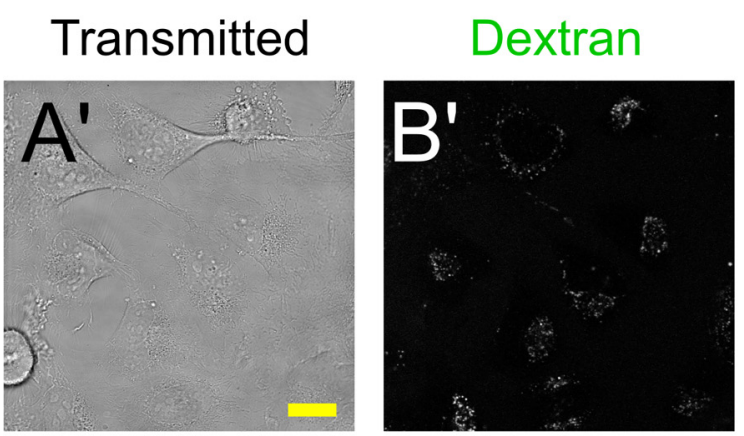

VEGF
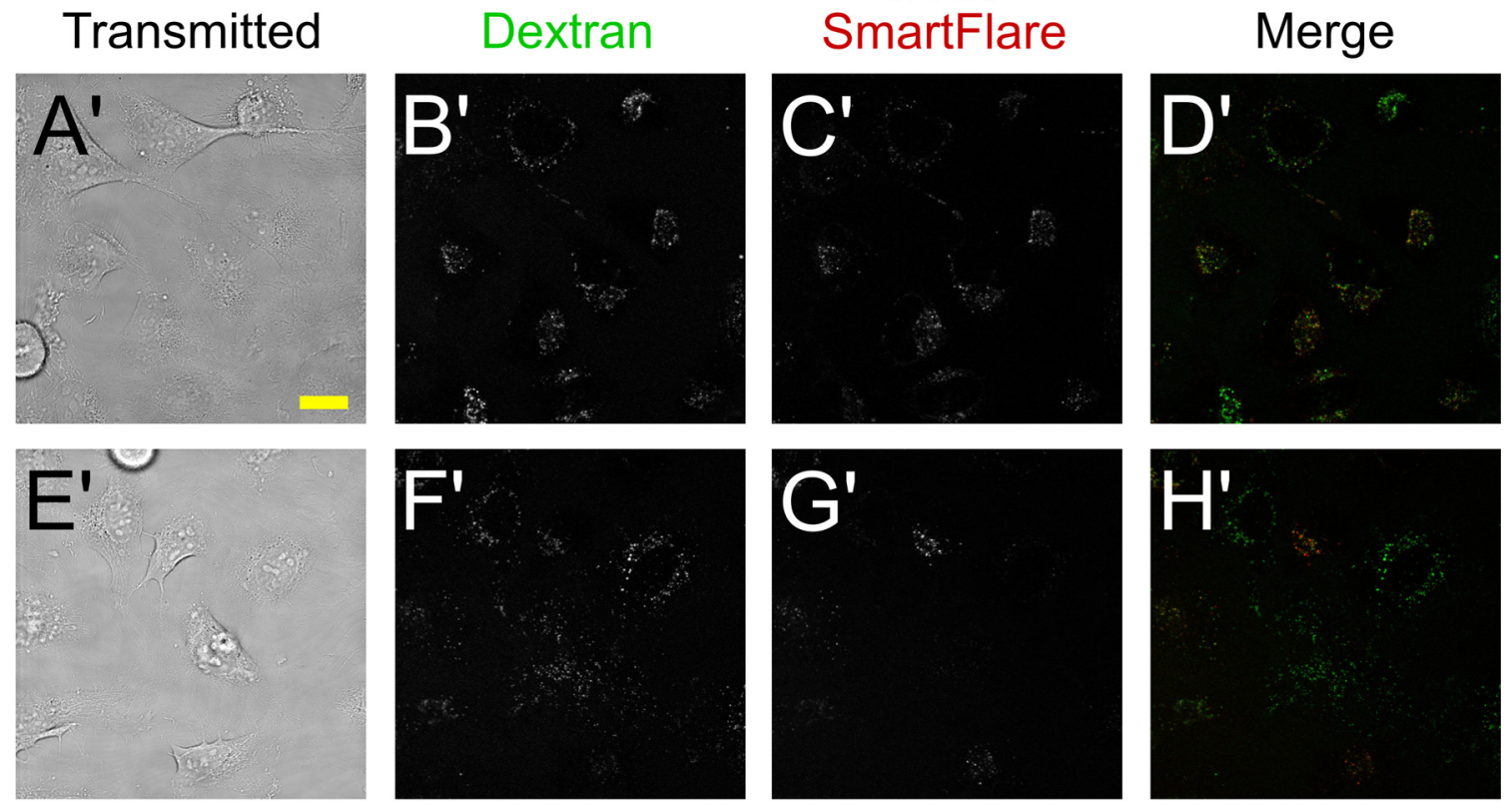

\section{Same field, imaged at best dynamic range}

Supplementary Figure S1. SmartFlare uptake at $\mathbf{2}$ hours: HeLa cells were exposed to Uptake Control (A-D) or VEGF (E-H) SmartFlares for 2 hours, then washed and imaged with confocal laser-scanning microscopy in fluorescence $(B, C, F, G)$ or transmitted $(A, E)$ channels. An overlay of the SmartFlares (pseudo-coloured red) and Dextran (pseudo-coloured green) is shown in $D \& H$. The top panels (A-H) show the same imaging parameters as were used for 18 hour experiments. The bottom panels $\left(A^{\prime}-H^{\prime}\right)$ shows the identical field imaged with optimal dynamic range for visualisation. All frames are shown at identical magnification, scale bar represents $20 \mu \mathrm{m}$. 